${ }^{1}$ Betriebsärztlicher Dienst, Klinikum der Johann Wolfgang Goethe-Universität Frankfurt

${ }^{2}$ Institut für Medizinische Virologie, Klinikum der Johann Wolfgang Goethe-Universität Frankfurt

${ }^{3}$ Institut für Arbeitsmedizin, Charité - Universitätsmedizin Berlin, Zentrum für Human- und Gesundheitswissenschaften, Freie Universität \& Humboldt-Universität zu Berlin

\title{
Arbeitsbedingte Infektionen bei Mitarbeitern des Gesundheitswesens: Respiratorische Erkrankungen
}

Sabine Wicker, Holger F. Rabenau, David A. Groneberg, René Gottschalk

S. Wicker, H.F. Rabenau, D.A. Groneberg, R. Gottschalk: Arbeitsbedingte Infektionen bei Mitarbeitern des Gesundheitswesens: Respiratorische Erkrankungen. Zbl Arbeitsmed 59 (2009) 82-91

Schlüsselwörter: aerogene Infektionen - arbeitsbedingte Infektionen - respiratorische Erkrankungen

Zusammenfassung:

Einführung: Mitarbeiter im Gesundheitswesen haben aufgrund ihrer Tätigkeit ein erhöhtes Infektionsrisiko gegenüber aerogen übertragbaren Erkrankungen. Zahlreiche Todesfälle sind im internationalen Umfeld beschrieben worden. Exemplarisch seien in diesem Kontext die Todesfälle während der SARS-Epidemie sowie Todesfälle durch Influenza genannt.

Methoden und Ergebnisse: Selektive Literaturaufarbeitung der Autoren zu potenziell aerogen übertragbaren Erregern wie SARS-Coronavirus, Influenzavirus, Parainfluenzavirus, RSV, Adenovirus, Tuberkulose sowie Pertussis. Die Literaturrecherche zeigt, dass sowohl durch persönliche Schutzmaßnahmen (z.B. Händehygiene, Tragen von Atemschutz) als auch durch Impfungen (sofern verfügbar) die Infektionsgefährdung der Mitarbeiter reduziert werden kann.

Diskussion: Das Risiko arbeitsbedingter Infektionen ist mitunter ein täglicher Bestandteil der Arbeit der Beschäftigten im Gesundheitswesen. Aus arbeitsmedizinischer und infektiologischer Sicht sollten Maßnahmen ergriffen werden, um den bestmöglichen Schutz der betroffenen Mitarbeiter zu gewährleisten. Dies ist gerade vor dem Hintergrund einer etwaigen Pandemie (z.B. Influenzapandemie) dringend erforderlich.

Occupationally acquired infections among health care workers: Respiratory diseases

S. Wicker, H.F. Rabenau, D.A. Groneberg, R. Gottschalk: Occupationally acquired infections among health care workers: Respiratory diseases. Zbl Arbeitsmed 59 (2009) 82-91

Key words: airborne transmission - occupational infectious diseases - respiratory diseases

Abstract:

Introduction: In some medical departments, healthcare workers (HCWs) are at risk for aerogene transmitted infectious diseases. Numerous fatal causalities were described in the international environment. For example fatal causalities during the SARS-Epidemic as well as cases of death caused by influenza.

Methods and results: Selective literature review of occupationally acquired aerogene infections like SARS-coronavirus, influenza virus, parainfluenza virus, RSV, adenovirus, tuberculosis as well as pertussis. Currently available data demonstrate that personal protective measures (e.g. appropriate use of hand washing and oronasal mask), as well as vaccinations (if available) reduces the risk for transmission of infectious diseases.

Discussion: The risk for occupationally acquired infections is sometimes an unavoidable part of daily patient care. From occupational medicine point of view and for control of infectious diseases preventive measures should be implemented to provide the best possible protection of HCW involved. With regard to a possible pandemic (e.g. influenza pandemic) this procedure is absolutely required.

Die Autoren:

Dr. med. Sabine Wicker ${ }^{1,2}$ - Prof. Dr. rer. med. Holger F. Rabenau ${ }^{2}$

Prof. Dr. med. Dr. h. c. David A. Groneberg 1 PD Dr. med. Dr. med. habil. René Gottschalk ${ }^{2}$

${ }_{2}^{1}$ Betriebsärztlicher Dienst $\square$ Klinikum der Johann Wolfgang Goethe-Universität Frankfurt $\square$ Theodor-Stern-Kai 7 @ 60590 Frankfurt am Main

2 Institut für Medizinische Virologie $\square$ Klinikum der Johann Wolfgang Goethe-Universität Frankfurt $\square$ Paul-Ehrlich-Str. 40 @0596 Frankfurt am Main

Institut für Arbeitsmedizin — Charité - Universitätsmedizin Berlin — Zentrum für Human- und Gesundheitswissenschaften $\mathbf{\square}$ Freie Universität \&

Humboldt-Universität zu Berlin —Thielallee 69-73 — 14195 Berlin

Korrespondenzanschrift:

Dr. Sabine Wicker $\boldsymbol{\square}$ Betriebsärztlicher Dienst Klinikum der Johann Wolfgang Goethe-Universität $\mathbf{\square}$ Theodor-Stern-Kai 7 - 60590 Frankfurt am Main Germany Telefon: +49(0)69-63014511—Facsimile: +49(0)69-63016385-E-Mail: Sabine.Wicker@kgu.de 
Nosokomiale respiratorische Infektionen sind sowohl für die Patienten als auch für die Mitarbeiter des Gesundheitswesens eine ernstzunehmende Gefährdung. Die im Jahr 2002/2003 gewonnenen Erfahrungen mit dem schweren akuten respiratorischen Syndrom (SARS) zeigen, dass das medizinische Personal einer im Vergleich zu der Allgemeinbevölkerung erhöhten Infektionsgefahr durch aerogen übertragbare Erreger ausgesetzt ist (Gottschalk 2005). Arbeitsbedingte aerogene Infektionsausbrüche sind häufig mit der substanziellen Verletzung einer oder mehrerer der drei grundlegenden Prinzipien der Krankenhaushygiene verbunden: Händedesinfektion, nicht geimpfte Beschäftigte des Gesundheitswesens sowie inadäquate und verzögerte Isolierung der potenziell infektiösen Patienten (Sepkowitz 1996; Seto et al. 2003).

Um Infektionsübertragungen auf Patienten $\mathrm{zu}$ vermeiden, sollten Mitarbeiter des Gesundheitswesens, die an einer akuten Infektion des Respirationstraktes erkrankt sind, keine immuninkompetenten Patienten (bspw. onkologische Patienten, Früh- und Neugeborene) betreuen (Tablan et al. 2004).

\section{Virale Infektionen}

1.1 Schweres akutes respiratorisches Syndrom (severe acute respiratory syndrom, SARS)

Da das SARS-assoziierte-Coronavirus (SARS-CoV) - Familie: Coronaviridae (siehe Abbildung 1) im Anfangsstadium mitunter grippeähnliche Symptome hervorruft und daher möglicherweise nicht frühzeitig genug erkannt werden kann, wären - im Falle eines Neuauftretens dieses Virus - Beschäftigte in Einrichtungen des Gesundheitswesens einem erhöhten Infektionsrisiko ausgesetzt. Etwaige protektive Maßnahmen würden durch eine verzögerte Diagnose nicht zeitgerecht ergriffen (z.B. adäquater Atemschutz FFP2/FFP3-Masken, Isolierung der betroffenen Patienten).

Die Inkubationszeit beträgt im Mittel 5 Tage, mit einer Zeitspanne von 2 bis 10 Tagen, wobei vereinzelte Berichte über Fälle mit längerer Inkubationszeit vorliegen. Das SARS-CoV wird im Rachensekret einige Tage (6-10 Tage) ausgeschieden, im Stuhl gelingt der RNA-

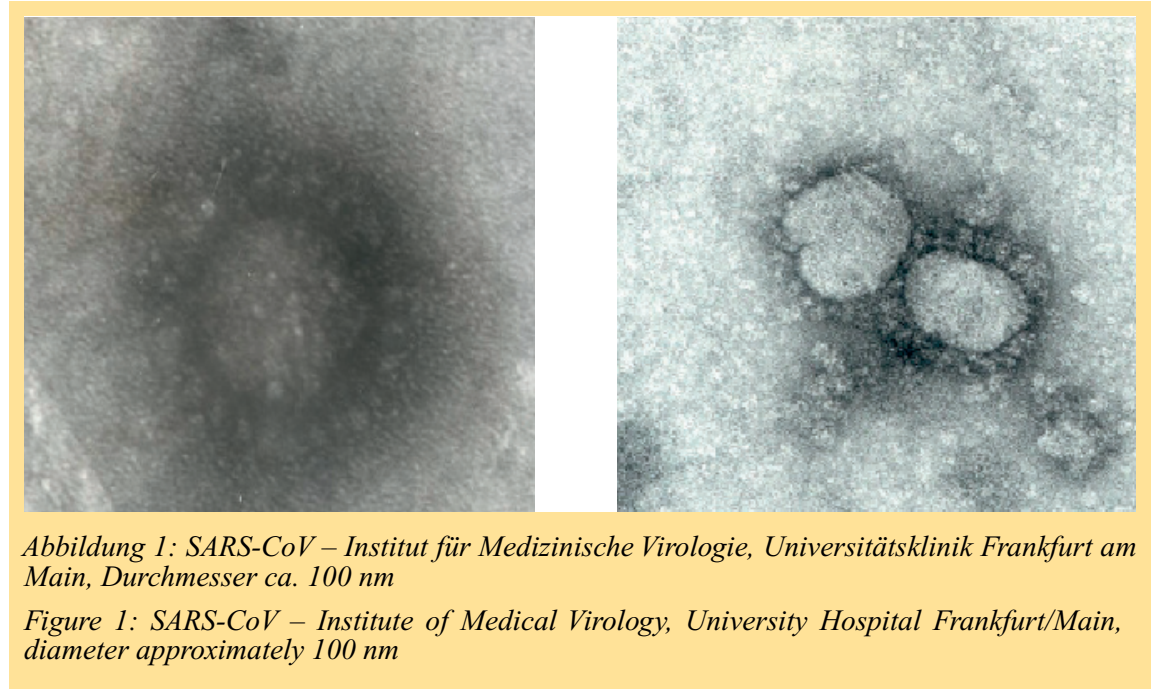

Nachweis noch bis zu 25 Tage, in Einzelfällen auch länger nach der Infektion. Als behülltes Virus ist SARS-CoV gegenüber Desinfektionsmitteln (mit dem Wirkspektrum „,begrenzt viruzid“") empfindlich (Rabenau et al. 2005a, 2005b). Der Erregernachweis ist dem Gesundheitsamt zu melden.

Im Zeitraum vom 1. November 2002 bis zum 31. Juli 2003 wurden insgesamt 8096 mögliche Fälle von SARS mit 774 Toten in 29 Ländern registriert (Pletz et al. 2007). Insgesamt handelte es sich bei $21 \%$ der SARS-Patienten um Beschäftigte im Gesundheitswesen, in einigen Ländern wie Kanada und Singapur waren es über $40 \%$ (Lenz et al. 2005). In einem Zeitraum von nur zwei Wochen nach dem Auftreten des Indexpatienten in Hongkong wurden 138 weitere Personen mit SARS-CoV infiziert. Die schnelle Ausbreitung des Virus von Patienten auf das Krankenhauspersonal sowie die Existenz von hyperinfektiösen Patienten (engl. ,super-spreader") weisen auf die hohe Infektiosität des Virus hin (Pletz et al. 2007). In einer Studie von 339 SARS-Fällen bei Mitarbeitern in 16 Krankenhäusern lag die Infektionsrate beim Pflegepersonal bei 1,2\% (Range 0-4,7\%), bei medizinisch-technischem Personal bei 0,3\% (Range $0-1,5 \%$ ) und beim medizinischen Hilfspersonal bei 2,7\% (0-13,3\%) (Lau et al. 2004). Eine Studie der Universitätsklinik Peking zeigte, dass Krankenpflegehelfer, welche besonders häufigen Kontakt mit den SARS-Patienten gehabt hatten, die höchste Infektionsrate aufwiesen $(6,7 \%)$, gefolgt von Krankenschwestern (4,8\%) und Ärzten (2,9\%). Nach der Einführung von persönlichen Schutzmaßnahmen und speziellen Stationen zur Behandlung von SARS-Patienten traten keine weitere Ansteckungen mehr auf(Li et al. 2003; Lenz et al. 2005).

Seto et al. konnten zeigen, dass es bei konsequenter Benutzung von persönlicher Schutzausrüstung (Schutzmaske, Überkittel, Handschuhe, Schutzbrille) und der Einhaltung von Hygienemaßnahmen (Händewaschen, Händedesinfektion) zu keinen Übertragungen auf das medizinische Personal gekommen war, wohingegen Infektionen bei Nichtbeachtung dieser Maßnahmen dokumentiert werden konnten (Seto et al. 2003).

Die Gefahr einer SARS-Pandemie ist jedoch vermutlich geringer als die einer Influenza-Pandemie, da mit InfluenzaViren infizierte Personen das Virus bereits vor der Manifestation einer Symptomatik ausscheiden, dagegen sind SARS-Patienten erst nach Ausbildung der klinischen Symptomatik infektiös (Pletz et al. 2007).

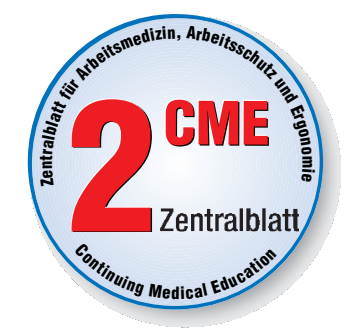




\subsection{Influenza-Viren}

(Familie: Orthomyxoviridae)

Influenzainfektionen (Typ A und B) treten weltweit sowohl epidemisch (sog. saisonale Grippewelle) als auch sporadisch als Pandemie auf, sie führen zu akuten, meist selbstlimitierenden Infektionen der oberen und unteren Luftwege. Bei Influenza A-Virus-Infektionen beruhen solche Epidemien vor allem auf der Möglichkeit, dass durch einzelne Punktmutationen (sogenannter Gen-Drift) oder den Austausch von Gensegmenten (sogenannter Gen-Shift) die Viren auf ein unzureichend bzw. nicht vorbereitetes Immunsystem beim Wirt (Mensch) trifft. Als Komplikation besonders der Influenza-A-Virus-Infektion kann eine primäre virale (z.T. schwere hämorrhagische Tracheobronchitis) oder sekundär eine bakterielle Pneumonie auftreten.

Die Inkubationszeit der Influenzainfektion beträgt 1-3 Tage. Das Influenzavirus (siehe Abbildung 2) wird von den Infizierten über das Rachensekret schon 24 Stunden vor Auftreten der ersten klinischen Symptome ausgeschieden, bei Kindern auch bis zu 48 Stunden vorher. Die Symptome manifestieren sich häufig als ,,sudden onset“" innerhalb von Minuten bis wenigen Stunden. Eine Virusausscheidung findet sich bei Erwachsenen und älteren Kindern für ca. 3-5 Tage, bei Säuglingen und Kleinkindern für ca. 1-2 Wochen und bei immunsupprimierten Patienten u.U. Wochen bis Monate. Auf Grund der hohen Variation der Influenzaviren sind wiederholte Infektionen und Erkrankungen im Laufe des Lebens möglich.

Medizinisches Personal hat einerseits ein erhöhtes Risiko, selbst an Influenza zu erkranken (Hallauer et al. 2005), andererseits sollen Patienten vor Übertragungen von Influenzaviren durch medizinisches Personal geschützt werden.

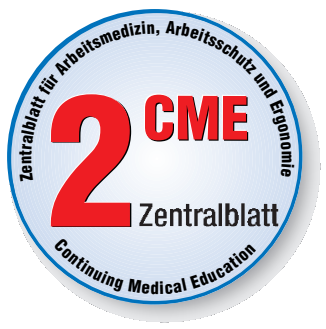

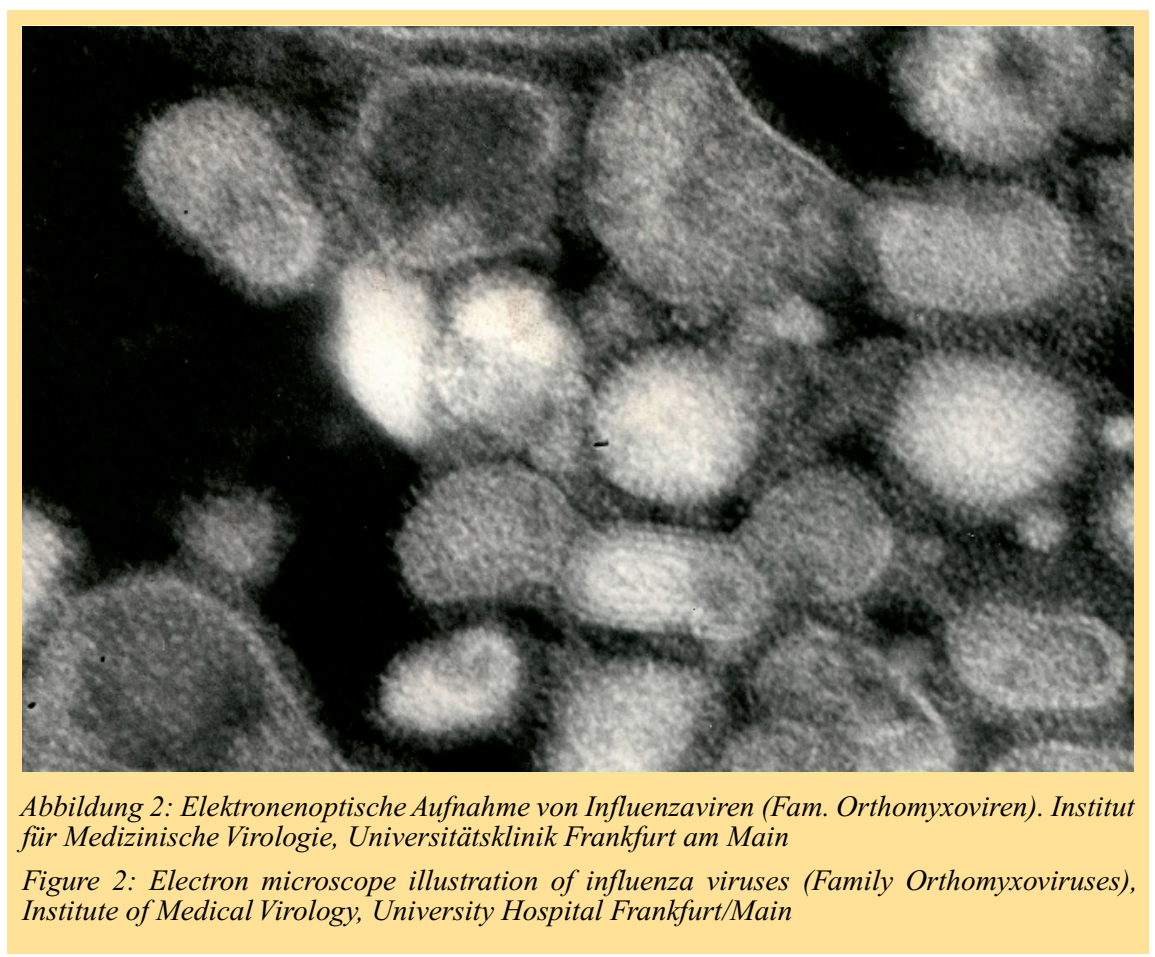

Nosokomiale Influenza-Ausbrüche wurden wiederholt in der Literatur beschrieben, beispielsweise in einer pädiatrischen Abteilung in New York, in der nosokomial erworbene Influenza-Viren bei 9,5\% der suszeptiblen Kinder nachgewiesen werden konnten (Hall 2007). In Deutschland ist der Erregernachweis nach $\S 7$ Infektionsschutzgesetz (IfSG) namentlich meldepflichtig.

Schätzungsweise $25 \%$ der ungeimpften Mitarbeiter des Gesundheitswesens infizieren sich mit Influenza (Hofmann et al. 2006). Allerdings zeigen nur 30\% der Mitarbeiter mit serologischem Nachweis einer Influenza-Infektion Influenzatypische Krankheitssymptome. Sowohl symptomatisch Erkrankte als auch asymptomatische Mitarbeiter können als Überträger fungieren (Elder et al. 1996).

Als behüllte Viren sind Influenzaviren gegenüber gängigen Desinfektionsmitteln (mit dem Wirkspektrum „,begrenzt viruzid") empfindlich.

Die Ständige Impfkommission (STIKO) benennt medizinisches Personal ausdrücklich als eine Risikogruppe, die gegen Influenza geimpft werden sollte. Vorrangig sollen die Patienten vor der Übertragung von Influenzaviren durch Pflege- und ärztliches Personal geschützt werden. Die routinemäßige Impfung des
Personals kann in Jahren mit einer starken Grippewelle die Sterblichkeit der betreuten Patienten deutlich senken (Hayward et al. 2006). Die Impfrate gegen Influenza ist jedoch bei medizinischem Personal in Deutschland mit meist deutlich unter 30\% nach wie vor zu niedrig (Wicker et al. 2007). Sie liegt deutlich unter denen der USA, wo durch Influenzaimpfprogramme beispielsweise in der Mayo Clinic Impfraten von $76,5 \%$ (Ofstead et al. 2008) und von $66 \%$ an der Universitätsklinik in Iowa erreicht wurden (Kuntz et al. 2008).

\subsection{Parainfluenza-Viren}

(Familie: Paramyxoviridae)

Eine Übertragung der Parainfluenzaviren (Typ 1-4) erfolgt durch Tröpfcheninfektionen, Aerosole, ggf. auch durch direkten Kontakt. Die Immunität nach überstandener Infektion ist nur kurzdauernd (ca. 3 Monate), sodass es immer wieder zu Reinfektionen kommen kann. Bei Erwachsenen kommt es meist nur zum „banalen Infekt“, wohingegen es bei Kindern bis 6 Jahre zu Laryngitis subglottica (Pseudokrupp), Rhinitis, Pharyngitis, Tracheobronchitis, Bronchiolitis oder Pneumonien kommen kann. Bei immuninkompetenten Patienten können schwere interstitielle Pneu- 
monien auftreten. Die Morbidität und Mortalität nach Knochenmarkstransplantationen können durch Parainfluenzavirusinfektionen ansteigen (Nichols et al. 2004). Die Inkubationszeit beträgt 3-6 Tage. Die Virusausscheidungsdauer über den Rachen beträgt bis 14 Tage (bei Infektion mit dem Typ 3 bis zu 4 Wochen).

Parainfluenzavirusübertragungen auf Mitarbeiter des Pflegepersonals eines Kinderkrankenhauses in Washington wurden beschrieben (Singh-Naz et al. 1990).

Spezifische Impfungen oder antivirale Substanzen stehen zum jetzigen Zeitpunkt nicht zur Verfügung. Bei Infektionen im Krankenhaus sollten konsequente Desinfektions- und Standardhygienemaßnahmen ergriffen werden (Händehygiene, Tragen von Handschuhen, Schutzbrillen, Schutzmasken und Schutzkittel, Patientenisolation). Als behüllte Viren sind Parainfluenzaviren gegenüber gängigen Desinfektionsmitteln (mit dem Wirkspektrum „,begrenzt viruzid“) empfindlich. Eine Meldpflicht nach IfSG besteht nicht.

\subsection{Respiratory Syncytial Viren \\ (Humanes Respiratorisches Synzytial-Virus, RS-Viren)}

Humane RS-Viren (Typ A und B - Familie: Paramyxoviridae) stellen eine wichtige Ursache von Pneumonien bei Säuglingen und Kleinkindern dar (Leidy et al. 2005). Sie verursachen aber auch sonstige Infekte der oberen Luftwege wie Rhinitis und Bronchitis und Otitis media.

Nosokomiale Infektionen weisen eine Mortalität bis 2\% auf, die Mortalität ist bei unterernährten Säuglingen und bei Kindern deutlich höher (bis zu 35\%). Die Inkubationszeit beträgt 3-7 Tage, eine Virusausscheidung kann bis zu 3 Wochen beobachtet werden, die Übertragung erfolgt durch Tröpfchen-, Aerosol- und Schmierinfektion. Wiederholte Infektionen auch mit Viren der gleichen Untergruppe sind häufig, d.h. es entsteht keine anhaltende Immunität. Zunehmend werden Infektionen bei älteren Patienten und (Knochenmark-/Stammzell-) Transplantierten beobachtet, infizierte Mitarbeiter können als Überträger fungieren (Liebert 2003; in: Adam, Doerr, Link, Lode). Bei immunkompetenten Erwachsenen verläuft eine RS-Infektion meist wie eine „banale Erkältung“. Eine Meldpflicht nach IfSG besteht nicht.

RSV besitzt eine relativ langandauernde Umweltstabilität, die Infektiosität kann in respiratorischem Sekret bis zu 20 Minuten auf den Händen erhalten bleiben, bis zu 45 Minuten auf Papierhandtüchern, bis zu 5 Stunden auf Einmalhandschuhen und bis zu 7 Stunden auf Kunststoffoberflächen (Hall et al. 1980). Das behüllte RSV ist jedoch gegenüber Desinfektionsmittel mit dem Wirkspektrum „,begrenzt viruzid“ sehr empfindlich. Ein Impfstoff steht zum jetzigen Zeitpunkt nicht zur Verfügung.

\subsection{Humanes Adenovirus \\ (Familie: Adenoviridae)}

Humane Adenoviren werden in Spezies $\mathrm{A}-\mathrm{F}$ eingeteilt, die insgesamt 51 Serotypen umfassen. Adenovirus-Infektionen sind weltweit verbreitet und für ca. 5\% der akuten Infektionen des oberen und unteren Respirationstraktes verantwortlich. Die Übertragung von Adenoviren erfolgt durch Tröpfchen- oder Schmierinfektionen. Die Inkubationszeit bei Atemwegsinfektionen beträgt 2-6 Tage, die Ausscheidungsdauer über den Rachen ca. 2-5 Tage, bei generalisiertem respiratorischem Infekt 3-6 Wochen und bei immunsupprimierten $\mathrm{Pa}$ tienten 2 bis 12 Monate. Adenoviruspneumonien beim Kleinkind können auch ohne Superinfektion schwer verlaufen. Auch bei Immunsupprimierten (z.B. Knochenmarkstransplantierten) spielen Adenoviren eine bedeutende Rolle. Eine Infektion mit Adenoviren (häufig im frühen Kindesalter) hinterlässt eine serotypspezifische Immunität, die allerdings nicht vor Reinfektion schützt, aber weitere Erkrankungen mit anderen Typen abmildern soll.

Bei nosokomialen Infektionen sollten umgehend Hygienemaßnahmen eingeleitet werden. Zum Schutz vor respiratorisch übertragenen Adenoviren sind Mund-/ Nasenschutz anzuwenden und nach Kontakt die Hände ordnungsgemäß zu desinfizieren. Dabei ist zu berücksichtigen, dass Adenoviren auch über unbelebte Vektoren (z.B. Türgriffe, Handläufe, Lichtschalter) übertragen werden können. Als nicht-behüllte Viren sind Adenoviren relativ bis sehr stabil gegen- über Desinfektionsmitteln. Daher sind im Verdachtsfall Hände- bzw. Flächendesinfektionsmittel mit dem Wirkspektrum ,viruzid“ zu verwenden.

Eine weitere große Gruppe der durch Adenoviren verursachten Erkrankungen sind Infekte des Gastrointestinaltraktes sowie der Augen. Die okkulären Adenovirusinfektionen stellen ein großes Problem für Augenkliniken und ophthalmologische Praxen dar. Der Erregernachweis ist nur im Konjunktivalabstrich namentlich meldepflichtig (§ 7 IfSG).

\subsection{Andere Virusinfektionen:}

Darüber hinaus existieren noch eine Reihe anderer Viren, die ein breites Bild respiratorischer oder pulmonaler Erkrankungen hervorrufen können. Exemplarisch seien in diesem Kontext Rhinovirus-, Varizellen-, Masern-, CMV- und Metapneumonievirus-Infektionen genannt.

\section{Bakterielle Infektionen}

\subsection{Tuberkulose (TB)}

Nach Schätzungen der Weltgesundheitsorganisation (WHO) erkranken weltweit jährlich 8-9 Millionen Menschen neu an Tuberkulose, schätzungsweise 2 Millionen Menschen sterben an den Folgen der Erkrankung. Erreger der Tuberkulose ist Mycobacterium tuberculosis, ein sporenbildendes säure- und alkoholfestes stäbchenförmiges Bakterium. Die Entstehung von multiresistenten Tuberkulosebakterien (MDR) sowie von so genannten extrem resistenten Tuberkulosestämmen (XDR) ist besorgniserregend, da diese hochresistenten Tuberkulosestämme medikamentös kaum noch behandelbar sind. Anfang 2005 wurde in der südafrikanischen Provinz KwaZulu/ Natal diese bisher unbekannte Form der Tuberkulose entdeckt. Ihr Ursprung liegt in einem Krankenhaus der Region, von den dort 53 erkrankten Personen, darunter auch medizinisches Personal, starben 52 an der XDR-TB (durchschnittlich 16 Tage nach der Erstuntersuchung) (RKI 2007).

Im Jahr 2005 gab es schätzungsweise 424000 MDR-TB-Infektionen sowie 27000 XDR-TB Erkrankungen (Goldman et al. 2007). Solche XDR-Stämme wurden bereits in allen Teilen der Welt nachgewiesen, einige Fälle auch in Deutsch- 
land. Wobei es sich in Deutschland ausnahmslos um importierte Fälle handelte. Aus diesem Grunde sind sowohl Maßnahmen des öffentlichen Gesundheitswesens als auch krankenhaushygienische Regelungen erforderlich, um die weitere Ausbreitung dieser extensiv resistenten Tuberkulosestämme zu verhindern (Uplekar \& Lönnroth 2007).

Die Tuberkulose wird meist als Tröpfcheninfektion übertragen, diese führt i.d.R. zu einem primären Befall der Lunge (Primärkomplex). Nach 3-4 Wochen tritt dann eine zellvermittelte Immunantwort ein, Makrophagen können die TB-Bakterien zwar phagozytieren, aber nicht zerstören. Oft macht dieser Primärkomplex keine Symptome. Nach einer Latenzperiode kann es jedoch zur Reaktivierung kommen (v.a. bei Abwehrschwäche und schlechtem Allgemeinzustand oder Immunsuppression). Der Erregernachweis ist nach $§ 7$ IfSG namentlich meldepflichtig.

Arbeitsbedingte Infektionen von Mitarbeitern des Gesundheitswesens sind sowohl im nationalen als auch internationalen Umfeld wiederholt beschrieben worden. Neben dem pulmonalen Übertragungsweg wurden auch Fälle von Tuberkulose durch Nadelstichverletzungen nachgewiesen.

Gemäß den Empfehlungen des ABAS (Beschluss Nr. 609 vom Oktober 2003; http://baua.de/prax/abas/besch609.htm) sollten als persönliche Schutzausrüstung im Umgang mit Patienten mit offener TB FFP2-Masken getragen werden, da sich hier im Hustenaerosol Tuberkulosebakterien befinden können. Auch die TRBA 250 sieht bei Erregern der Risikogruppe 3 im Abs. 4.3.4. FFP 2-Masken vor. Für TB sind Tuberkulosewirksame Desinfektionsmittel gemäß den Empfehlungen des RKI (Robert Koch-Institut) bzw. des Verbundes für angewandte Hygiene (VAH) einzusetzen. Eine sorgsame Händedesinfektion ist nach direktem Patientenkontakt, nach Kontakt mit erregerhaltigem Material und nach Kontakt mit kontaminierten Gegenständen zwingend notwendig. $\mathrm{Zu}$ beachten ist die verlängerte Einwirkzeit (1 Minute) der Desinfektionsmittel bei diesem Erreger.

\subsection{Pertussis/Keuchhusten}

Bordetella pertussis ist hochinfektiös und wird i.d.R. durch Tröpfcheninfektion übertragen. Die Inkubationszeit beträgt 7-10 Tage, die Infektiosität hält für ca. 6 Wochen an, sofern sie nicht zuvor durch eine adäquate antibiotische $\mathrm{Be}$ handlung unterbrochen wird. Eine Meldepflicht nach IfSG besteht nicht. Weder die Infektion mit $B$. pertussis noch die Impfung führt zur lebenslangen Immunität.

Die WHO geht davon aus, dass es durch Pertussisinfektionen zu jährlich circa 300000 Todesfällen und schätzungsweise knapp 40 Millionen Erkrankungsfällen kommt (WHO 2005). Erwachsene sind eine häufige Übertragungsquelle für ungeimpfte und teilgeimpfte Kinder. Keuchhusten-Infektionen bei Kindern können zu schwerwiegenden Verläufen und mitunter Todesfällen führen (Hewlett \& Edwards 2005). Die Erkrankung ist für Säuglinge, die etwa $10 \%$ der Betroffenen ausmachen, besonders gefährlich und durch die nicht selten im Krankheitsverlauf auftretende Apnoe mitunter lebensbedrohend (Todesrate 1,8\% für Neugeborne und Kinder $<2$ Monate; Hood et al. 2008).

Beschäftigte des Gesundheitswesens mit Kontakt zu Kindern haben einerseits ein erhöhtes Expositionsrisiko und können andererseits auch als Überträger fungieren (Plotkin 2005). Nosokomiale Pertussis-Übertragungen von Mitarbeitern des Gesundheitswesens auf Patienten wurden wiederholt beschrieben (Bryant et al. 2006; Alexander et al. 2008). Das Robert Koch-Institut nennt medizinisches Personal mit Kontakt zu Kindern ausdrücklich als eine Risikogruppe, die gegenüber Pertussis geimpft sein sollte (RKI 2007). Die Impfung der Mitarbeiter spielt eine entscheidende Rolle in der Verhinderung von nosokomialen Ausbrüchen (Zivna et al. 2007; Calugar et al. 2006).

Im Juni 2008 wurde der Fall einer 24-jährigen Mitarbeiterin aus Texas beschrieben, welche insgesamt 11 von 113 der von ihr betreuten Neugeborenen mit Pertussis infizierte (Infektionsrate 9,7\%). Die Mitarbeiterin war als Kind vollständig gegen Pertussis geimpft worden, scheinbar erfolgten jedoch keine weiteren Boosterimpfungen (Hood et al. 2008).

\subsection{Andere bakterielle Infektionen:}

Darüber hinaus existieren noch eine Reihe anderer Bakterien, die ein breites Bild respiratorischer oder pulmonaler Erkrankungen hervorrufen können. Exemplarisch seien in diesem Kontext Legionella pneumophila, Pseudomonas aeruginosa sowie bakterienähnliche Erreger wie Mycoplasma pneumoniae genannt.

\section{Fazit für die Praxis:}

In den Empfehlungen des Robert Koch-Institutes vom 14. Oktober 2005 wird auf einen Beschluss des Ausschusses für biologische Arbeitsstoffe (ABAS) verwiesen. Dieser Beschluss (Nr. 609 vom Oktober 2003; http://baua.de/prax/ abas/besch609.htm) befasst sich mit dem Arbeitsschutz beim Auftreten von pandemischer Influenza unter besonderer Berücksichtigung des Atemschutzes. In diesen Empfehlungen wird als wichtigste individualhygienische Maßnahme für medizinisches Personal das Tragen eines dicht anliegenden Mund-Nasenschutzes empfohlen. Bei Tätigkeiten, bei denen medizinisches Personal Patienten ambulant versorgt oder Verdachtsfälle pflegt, soll eine Maske getragen werden, die hinsichtlich der Gesamtleckage des Filter-Durchlassgrades mindestens den Anforderungen der Geräteklassen FFP 1 entspricht. Bei Tätigkeiten, bei denen die Beschäftigten Hustenstößen von $\mathrm{Pa}$ tienten ausgesetzt sein könnten, sollen FFP 2-Masken getragen werden. Wird jedoch das Husten des Patienten provoziert, z.B. während einer Bronchoskopie, Intubation oder beim Absaugen, sind, laut dieses Beschlusses, FFP 3Masken zu tragen.

Aus der klinischen Kenntnis heraus ist diese Unterteilung unserer Meinung nach jedoch kaum praktikabel und lässt sich lediglich bei gezielten Eingriffen, wie z.B. bei einer bevorstehenden Bronchoskopie, realisieren. Hinzu kommen die schwierige Lagerhaltung für drei verschiedene Maskentypen und der Preisnachteil beim Einkauf kleinerer Stückmengen. Aus pragmatischer Sicht ist daher die Verwendung von chirurgischen Masken (bspw. für Kontakt mit 
Patienten mit Parainfluenzavirus-bedingten oder anderen viralen Infektionen) zu empfehlen, sofern keine besondere Erregerexposition gegeben ist. Demgegenüber sind beim Umgang mit Patienten mit unbekannten Erregern oder intensivem Kontakt (z.B. nach provoziertem Husten oder während einer Bronchoskopie) FFP 3-Masken zu bevorzugen.

Die Tragedauer von Masken wird im Allgemeinen unterschätzt. Insbesondere chirurgische Masken können lange getragen werden, so dass man in aller Regel mit zwei Masken pro Tag und Mitarbeiter rechnen sollte.

Effektive Maßnahmen zur Infektionskontrolle sollten nicht dahingehend kommuniziert werden, dass spezifische Empfehlungen für jeweils einzelne Erreger entwickelt werden, stattdessen sollten adäquate Standardhygienemaßnahmen implementiert werden, die für die Mitarbeiter nachvollziehbar, praktikabel in der täglichen Arbeit und ebenso zumutbar sind. Diese Maßnahmen zur Infektionskontrolle sollten transparent publiziert und von allen unterschiedlichen Berufsgruppen stringent und einheitlich befolgt werden. Hierbei spielen Vorgesetzte im Sinne einer Vorbildfunktion eine besondere Rolle.

Für den Umgang mit Erkrankten mit hochpathogenen Erregern (z.B. SARS, Vogelgrippe) sollten die Mitarbeiter gesondert geschult werden.

\section{Literatur:}

Adam D, Doerr HW, Link H, Lode H (2003) Die Infektiologie. Springer Verlag Berlin, Heidelberg, New York

Alexander EM, Travis S, Booms C et al. (2008) Pertussis outbreak on a neonatal unit: identification of a healthcare worker as the likely source. J Hosp Infect 69: 131-134

Bryant KA, Humbaugh K, Brothers K et al. (2006) Measures to control an outbreak of pertussis in a neonatal intermediate care nursery after exposure to a healthcare worker. Infect Control Hosp Epidemiol 27: 541-545

Calugar A, Ortega-Sánchez Ir, Tiwari T, Oakes L, Jahre JA, Murphy TV (2006) Nosocomial pertussis: costs of an outbreak and benefits of vaccinating health care workers. Clin Infect Dis 42: 981-988

Elder AG, O'Donnell B, McCruden EAB, Symington IA, Carman WF (1996) Incidence and recall of influenza in a cohort of Glasgow HCWs during the 1993-4 epidemic: results of serum testing and questionnaire. BMJ 313: $1241-1242$
Goldman RC, Plumley KV, Laughon BE (2007) The evolution of extensively drug resistant tuberculosis (XDR-TB): history, status and issues for global control. Infect Disord Drug Targets 2: 73-91

Gottschalk R (2005) Neue und hochinfektiöse Krankheitserreger - Entwicklung von Maßnahmen der Seuchenabwehr durch den Offentlichen Gesundheitsdienst am Beispiel SARS Verlag der Akademie für öffentliches Gesundheitswesen, Düsseldorf

Hall CB (2007) The Spread of Influenza and Other Respiratory Viruses. Complexities and Conjectures. CID 45: 353-359

Hall CB, Douglas RG Jr, Geiman JM (1980) Possible transmission by fomites of respiratory syncytial virus. J Infect Dis 141: 98-102

Hallauer JF, Neuschaefer-Rube N (2005) Influenza vaccination of hospital staff in Germany: a five-year survey on vaccination coverage and policies: identified deficits in influenza immunisation campaigns for hospital employees. Soz Präventivmed 50: 38-44

Hayward AC, Harling R, Wetten S et al. (2006) Effectiveness of an influenza vaccine programme for care home staff to prevent death, morbidity, and health service use among residents: cluster randomised controlled trial. BMJ 333 (7581): 1229-1230

Hewlett EL, Edwards KM (2005) Pertussis not just for kids. N Engl J Med 352: 1215-1222

Hofmann F, Ferracin C, Marsh G, Dumas R (2006) Influenza vaccination of healthcare workers: a literature review of attitudes and beliefs. Infection 34: 142-147

Hood JL, Murphey DK, Dunn JJ (2008) Hospital-Acquired Pertussis among NewbornsTexas, 2004. MMWR 22: 600-603

Kuntz JL, Holley S, Helms CM, Cavanaugh JE, Berg JV, Herwaldt LA, Polgreen PM (2008) Use of a pandemic preparedness drill to increase rates of influenza vaccination among healthcare workers. Infect Control Hosp Epidemiol 29:111-115

Lau JT, Fung KS, Wong TW, Kim JH, Wong E, Chung S, Ho D, Chan LY, Lui SF, Cheng A (2004) SARS transmission among hospital workers in Hong Kong. Emerg Infect Dis 10:280-286

Leidy NK, Margolis MK, Marcin JP, Flynn JA, Frankel LR, Johnson S, Langkamp D, Simoes EA (2005) The impact of serve respiratory syncytial virus on the child, caregiver, and family during hospitalization and recovery. Pediatrics 115: 1536-1546

Lenz M, Groneberg DA, Schäcke G (2005) Schweres respiratorisches Syndrom - SARS in der Arbeits- und Umweltmedizin. Zbl Arbeitsmedizin 55: 254-262

Li L, Cheng S, Gu J (2003) SARS infection among health care workers in Beijing, China. JAMA 290: 662-2663

Nichols WG, Erdmann DD, Han A, Zukerman C, Corey L, Boeckh M (2004) Prolonged outbreak of human parainfluenza virus 3 infection in a stem cell transplant outpatient department: insights from molecular epidemiologic analysis. Biol Blood Marrow Transplant 10: 58-64 Ofstead CL, Tucker SJ, Beebe TJ, Polamd GA (2008) Influenza vaccination among registered nurses: Information receipt, knowledge, and decision-making at an institution with a multifaceted educational program. Infect Control Hosp Epidemiol 29:99-106
Pittet D, Allegranzi B, Sax H, Dharan S, PessoaSilva CL, Donaldson L, Boyce JM; WHO Global Patient Safety Challenge, World Alliance for Patient Safety (2006) Evidence-based model for hand transmission during patient care and the role of improved practices.Lancet Infect Dis. 10: 641-652

Pletz MW, Dickgreber N, Hagen L, Golpon H, Zabel P, Bauer TT, Welte T, Groneberg DA (2007) Impfstrategien bei Schwerem Akutem Respiratorischem Syndrom (SARS). Pneumologie 61: 663-668

Plotkin S (2005) The Global Pertussis Initiative. Pediatr Infect Dis J Vol 24 Supp

Rabenau HF, Cinatl J, Morgenstern B, Bauer G, Preiser W, Doerr HW (2005a) Stability and inactivation of SARS coronavirus. Med Microbiol Immunol 194: 1-6

Rabenau HF, Kampf G, Cinatl J, Doerr HW (2005b) Efficacy of various disinfectants against the SARS-Coronavirus (SARS-CoV). Hospital Infection 61(2): 107-111

Robert Koch Institute (RKI) http://www.rki.de

Robert Koch Institut (2007) Tuberkulose irgendwo heißt Tuberkulose überall. Epidemiologisches Bulletin 2007; 12: 87-91

Sepkowitz KA (1996) Occupationally acquired infections in health care workers. Part I. Ann Intern Med 125: 826-834

Seto WH, Tsang D, Yung RW, Ching TY, Ng TK Ho M, Ho LM, Peiris JS; Advisors of Expert SARS group of Hospital Authority (2003) Effectiveness of precautions against droplets and contact in prevention of nosocomial transmission of severe acute respiratory syndrome (SARS). Lancet 361: 1591-1520

Singh-Naz N, Willy M, Riggs N (1990) Outbreak of parainfluenza virus type 3 in a neonatal nursery. Pediatr Infect Dis J 31-33

Tablan OC, Anderson LJ, Besser R, Bridges C, Hajjeh R (2004) Guidelines for Preventing Health-Care Associated Pneumonia, 2003. Recommendations of CDC and the Healthcare Infection Control Practices Advisory Committee. MMWR 53: 1-36

Upleklar M, Lönnroth K (2007) MDR and XDR - price of delaying engagement with all care providers for control of TB and TB/HIV Trop Med Int Health 12: 473-474

Vernon MO, Trick WE, Welbel SF, Peterson BJ Weinstein RA (2003) Adherence with hand hygiene: does number of sinks matter? Infect Control Hosp Epidemiol 24: 224-225

World Health Organisation http://www.who.int/immunization/topics/ pertussis/en/print.html

Wicker S, Doerr HW, Gottschalk R, Rabenau HF, Allwinn R (2007) Influenza: Akzeptanz der Schutzimpfung bei medizinischem Personal Auswertung zur Influenzasaison 2006/2007. Dtsch Med Wochenschr 132: 1683-7

Zivna I, Bergin D, Casavant J, Fontecchio S, Nelson S, Kelley A, Mathis S, Melvin Z, Erlichman R, Ellison RT (2007) Impact of Bordetella pertussis exposures on a Massachusetts tertiary care medical system. Infect Control Hosp Epidemiol 28: 708-712 


\section{CME-Fragen: Respiratorische Erkrankungen}

\begin{tabular}{|c|c|c|c|}
\hline 1. & Wie verläuft eine SARS-Infektion beim Menschen? & 6. & Welche Aussage zur Bedeutung der Tuberkulose trifft zu? \\
\hline A & Lange Inkubationszeit (30-180 Tage), schleichender Beginn & A & $\begin{array}{l}\text { Durch die moderne Antibiotikatherapie hat die Tuberkulose an } \\
\text { Bedeutung verloren. }\end{array}$ \\
\hline B & Die Infektion heilt stets folgenlos aus. & $\mathrm{B}$ & Jährlich erkranken weltweit max. 2 Millionen Menschen an Tuberkulose. \\
\hline C & $\begin{array}{l}\text { Bereits 1-2 Wochen vor den ersten Symptomen wird das Virus } \\
\text { in hoher Konzentration im Rachensekret ausgeschieden. }\end{array}$ & $\mathrm{C}$ & Multiresistente Tuberkuloseformen treten in Deutschland nicht auf. \\
\hline $\mathrm{D}$ & $\begin{array}{l}\text { Das Tragen von persönlicher Schutzkleidung hat keinen Ein- } \\
\text { fluss auf die Infektionsübertragung auf medizinisches Personal. }\end{array}$ & $\mathrm{D}$ & $\begin{array}{l}\text { XDR-Stämme zeichnen sich durch eine gute Ansprechbarkeit auf die } \\
\text { gängigen Medikamente aus. }\end{array}$ \\
\hline $\mathrm{E}$ & Die Inkubationszeit beträgt im Mittel 5 Tage (Range 2-10 Tage). & $\mathrm{E}$ & $\begin{array}{l}\text { Der primäre Befall der Lunge (Primärkomplex) macht oft keine } \\
\text { Symptome. }\end{array}$ \\
\hline 2. & Was trifft zu? & 7. & $\begin{array}{l}\text { Infektionen durch B. pertussis können bei Kindern zu } \\
\text { schwerwiegenden Verläufen führen. Was trifft zu? }\end{array}$ \\
\hline A & $\begin{array}{l}\text { Eine Influenzainfektion bei einem ungeimpften Mitarbeiter des } \\
\text { Gesundheitswesens ist ein seltenes Ereignis. }\end{array}$ & A & Die Erkrankung ist v.a. für Säuglinge besonders gefährlich. \\
\hline B & $\begin{array}{l}\text { Die Influenzaimpfraten des medizinischen Personals in } \\
\text { Deutschland liegen bei über } 60 \% \text {. }\end{array}$ & B & Eine antibiotische Behandlung steht nicht zur Verfügung. \\
\hline C & $\begin{array}{l}\text { Die Influenzaimpfung des medizinischen Personals kann die } \\
\text { Sterblichkeit der betreuten Patienten senken. }\end{array}$ & $\mathrm{C}$ & Eine Pertussisinfektion hinterlässt eine lebenslange Immunität. \\
\hline $\mathrm{D}$ & Die Inkubationszeit beträgt 6-10 Tage. & $\mathrm{D}$ & $\begin{array}{l}\text { Medizinisches Personal spielt in der Übertragung von Pertussis keine } \\
\text { Rolle. }\end{array}$ \\
\hline $\mathrm{E}$ & $\begin{array}{l}\text { Das Influenzavirus wird erst } 24 \text { Stunden nach dem Auftreten } \\
\text { erster Symptome über das Rachensekret ausgeschieden. }\end{array}$ & $\mathrm{E}$ & $\begin{array}{l}\text { Eine Pertussis-Impfempfehlung für medizinisches Personal existiert } \\
\text { nicht. }\end{array}$ \\
\hline 3. & $\begin{array}{l}\text { Was trifft nicht zu? Arbeitsbedingte virale respiratorische } \\
\text { Infektionen können durch folgende Erreger verursacht } \\
\text { werden: }\end{array}$ & 8. & $\begin{array}{l}\text { Was trifft nicht zu? } \\
\text { Arbeitsbedingte bakterielle respiratorische Infektionen können } \\
\text { durch folgende Erreger verursacht werden: }\end{array}$ \\
\hline A & SARS & A & Bordetella pertussis \\
\hline B & Influenza & B & Mycobacterium tuberculosis \\
\hline C & Pertussis & $\mathrm{C}$ & Legionella pneumophila \\
\hline $\mathrm{D}$ & Parainfluenza & $\mathrm{D}$ & Pseudomonas aeruginosa \\
\hline $\mathrm{E}$ & Masern & $\mathrm{E}$ & Metapneumonievirus \\
\hline 4. & $\begin{array}{l}\text { Die Empfindlichkeit gegenüber den gängigen } \\
\text { Desinfektionsmitteln ist bei den verschiedenen Viren } \\
\text { unterschiedlich. Was trifft nicht zu? }\end{array}$ & 9. & $\begin{array}{l}\text { Atemschutz spielt bei der Vermeidung von aerogen übertragbaren } \\
\text { Erkrankungen eine wichtige Rolle. Welche Aussagen zu Atem- } \\
\text { schutzmasken treffen zu? }\end{array}$ \\
\hline A & $\begin{array}{l}\text { Als behüllte Viren sind Parainfluenzaviren gegenüber gängigen } \\
\text { Desinfektionsmitteln empfindlich. }\end{array}$ & A & $\begin{array}{l}\text { FFP 3-Masken sollten für alle Mitarbeiter eines Krankenhauses bei } \\
\text { Verdacht auf eine Influenzapandemie verwandt werden. }\end{array}$ \\
\hline B & $\begin{array}{l}\text { Das behüllte RSV ist gegenüber Desinfektionsmitteln nicht } \\
\text { empfindlich. }\end{array}$ & B & $\begin{array}{l}\text { Das Tragen von chirurgischen MNS bedingt eine Untersuchungspflicht } \\
\text { nach G26. }\end{array}$ \\
\hline $\mathrm{C}$ & $\begin{array}{l}\text { Als behülltes Virus ist SARS-CoV gegenüber Desinfektions- } \\
\text { mitteln empfindlich. }\end{array}$ & $\mathrm{C}$ & FFP 1 Masken weisen die geringste Gesamtleckage auf. \\
\hline $\mathrm{D}$ & $\begin{array}{l}\text { Als behülte Viren sind Influenzaviren gegenüber gängigen } \\
\text { Desinfektionsmitteln empfindlich. }\end{array}$ & $\mathrm{D}$ & $\begin{array}{l}\text { Bei der Bronchoskopie eines an SARS verdächtigten Patienten sollte } \\
\text { eine FFP } 1 \text { Maske getragen werden. }\end{array}$ \\
\hline $\mathrm{E}$ & $\begin{array}{l}\text { Als nicht-behüllte Viren sind Adenoviren relativ bis sehr stabil } \\
\text { gegenüber Desinfektionsmitteln. }\end{array}$ & $\mathrm{E}$ & $\begin{array}{l}\text { Für den Umgang mit Erkrankten mit hochpathogenen Erregern sollten } \\
\text { die Mitarbeiter gesondert geschult werden. }\end{array}$ \\
\hline 5. & $\begin{array}{l}\text { Immunsupprimierte Patienten sind durch nosokomiale } \\
\text { Infektionen besonders gefährdet. Schwerwiegende } \\
\text { respiratorische Erkrankungen werden häufig durch folgen- } \\
\text { de Erreger verursacht (s.u.) Was trifft nicht zu? }\end{array}$ & 10. & $\begin{array}{l}\text { Medizinisches Personal ist einer im Vergleich zur Allgemeinbevölke- } \\
\text { rung erhöhten Infektionsgefährdung ausgesetzt. Folgende Maßnah- } \\
\text { men können die Infektionsgefahr der Beschäftigten minimieren: } \\
\text { 1. Impfungen } \\
\text { 2. Maßnahmen der Händehygiene } \\
\text { 3. Tragen eines adäquaten Mundschutzes } \\
\text { 4. Isolierung der potenziell infektiösen Patienten } \\
\text { 5. Verwendung von auf ihre Wirksamkeit hin überprüften } \\
\text { Desinfektionsmitteln }\end{array}$ \\
\hline A & CMV & A & 1 und 2 ist richtig \\
\hline B & Parainfluenza & $\mathrm{B}$ & $1,2,3$ und 5 sind richtig \\
\hline $\mathrm{C}$ & Influenza & $\mathrm{C}$ & $2,3,4$ sind richtig \\
\hline $\mathrm{D}$ & E. coli & $\mathrm{D}$ & 1 und 4 sind richtig \\
\hline $\mathrm{E}$ & Respiratory Syncytial Viren & $\mathrm{E}$ & alle sind richtig \\
\hline
\end{tabular}




\section{CME-Fragen: Respiratorische Erkrankungen}

\begin{tabular}{|c|c|c|c|}
\hline 11. & $\begin{array}{l}\text { Als Komplikation der Influenza können folgende } \\
\text { Erkrankungen auftreten: } \\
\text { 1. Allergischer Schock } \\
\text { 2. Schwere hämorrhagische Tracheobronchitis } \\
\text { 3. Bakterielle Pneumonien } \\
\text { 4. Hautmykosen } \\
\text { 5. Pruritus }\end{array}$ & 16. & Welche Angabe zu Keuchhusten ist korrekt? \\
\hline A & 1 und 2 ist richtig & A & Keuchhusten wird durch Pseudomonas aeruginosa verursacht. \\
\hline B & $1,2,3$ und 5 sind richtig & $\mathrm{B}$ & Keuchhusten wird durch Klebsiella pneumoniae verursacht. \\
\hline $\mathrm{C}$ & 2 und 3 sind richtig & $\mathrm{C}$ & Keuchhusten wird durch Mycoplasma pneumoniae verursacht. \\
\hline $\mathrm{D}$ & 1 und 4 sind richtig & $\mathrm{D}$ & Keuchhusten wird durch Parainfluenzaviren verursacht. \\
\hline $\mathrm{E}$ & alle sind richtig & $\mathrm{E}$ & Keuchhusten wird durch Bordetella pertussis verursacht. \\
\hline 12. & $\begin{array}{l}\text { Was trifft zu? } \\
\text { 1. Medizinisches Personal hat ein erhöhtes Risiko an Influenza } \\
\text { zu erkranken. } \\
\text { 2. Medizinisches Personal hat ein verringertes Risiko an } \\
\text { Influenza zu erkranken. } \\
\text { 3. Medizinisches Personal hat ein ebenso hohes Risiko an } \\
\text { Influenza zu erkranken wie die Normalbevölkerung. } \\
\text { 4. Die Impfung führt bei weniger als } 20 \% \text { der Geimpften zu } \\
\text { einem hinreichenden Schutz. } \\
\text { 5. Die Impfung führt bei mehr als } 80 \% \text { der Geimpften zu einem } \\
\text { hinreichenden Schutz. }\end{array}$ & 17. & Welche Aussage zu Bordetella pertussis trifft zu? \\
\hline A & 1 und 4 ist richtig & A & Der Erreger wird meist parenteral übertragen. \\
\hline B & 3 und 5 sind richtig & $\mathrm{B}$ & Die Inkubationszeit beträgt 14-30 Tage. \\
\hline $\mathrm{C}$ & 2 und 4 sind richtig & $\mathrm{C}$ & $\begin{array}{l}\text { Die Infektiosität hält für ca. } 6 \text { Wochen an, sofern sie nicht zuvor durch } \\
\text { eine adäquate antibiotische Behandlung unterbrochen wird. }\end{array}$ \\
\hline $\mathrm{D}$ & 1 und 4 sind richtig & $\mathrm{D}$ & Die Impfung hinterlässt meist eine lebenslange Immunität. \\
\hline E & 1 und 5 sind richtig & $\mathrm{E}$ & Nosokomiale Übertragungen spielen keine Rolle. \\
\hline 13. & Welche Angabe zu Parainfluenza ist korrekt? & 18. & Welche Aussage zu Bordetella pertussis trifft nicht zu? \\
\hline A & Es existierten Parainfluenza A, B und C-Virustypen. & A & Bordetella pertussis ist hochinfektiös. \\
\hline B & Man unterscheidet Parainfluenza Typ 1-4. & $\mathrm{B}$ & Eine Meldepflicht nach IfSG besteht nicht. \\
\hline C & $\begin{array}{l}\text { Bei Kindern verläuft die Erkrankung leichter als bei } \\
\text { Erwachsenen. }\end{array}$ & $\mathrm{C}$ & $\begin{array}{l}\text { Die WHO geht davon aus, dass es weltweit zu jährlich } 300.000 \text { Todes- } \\
\text { fällen durch Pertussisinfektionen kommt. }\end{array}$ \\
\hline $\mathrm{D}$ & $\begin{array}{l}\text { Immuninkompetente Patienten sind durch Parainfluenza- } \\
\text { infektionen nicht gefährdet. }\end{array}$ & $\mathrm{D}$ & $\begin{array}{l}\text { Die WHO geht davon aus, dass es weltweit zu jährlich } 40 \text { Millionen } \\
\text { Erkrankungsfällen durch Pertussisinfektionen kommt. }\end{array}$ \\
\hline $\mathrm{E}$ & $\begin{array}{l}\text { Die Morbidität und Mortalität nach Knochenmarkstransplanta- } \\
\text { tion wird durch Parainfluenzainfektionen nicht erhöht. }\end{array}$ & $\mathrm{E}$ & $\begin{array}{l}\text { Die Erkrankung ist für Säuglinge nicht gefährlich, da diese noch } \\
\text { genügend maternale Antikörper besitzen. }\end{array}$ \\
\hline 14. & Welche Angabe zu Respiratory Syncytial Viren ist korrekt? & 19. & Welche Aussage zur Tuberkulose trifft zu? \\
\hline A & $\begin{array}{l}\text { RS-Viren stellen eine wichtige Ursache von Pneumonien bei } \\
\text { Säuglingen und Kleinkindern dar. }\end{array}$ & A & $\begin{array}{l}\text { Weltweit sterben jährlich schätzungsweise 8-9 Millionen Menschen an } \\
\text { Tuberkulose. }\end{array}$ \\
\hline B & $\begin{array}{l}\text { Nosokomiale Infektionen weisen eine Mortalität von bis zu } \\
90 \% \text { auf. }\end{array}$ & $\mathrm{B}$ & $\begin{array}{l}\text { Mycobacterium tuberculosis gehört zu den säure- und stäbchen- } \\
\text { förmigen Viren. }\end{array}$ \\
\hline $\mathrm{C}$ & Die Inkubationszeit beträgt 14 Tage. & $\mathrm{C}$ & $\begin{array}{l}\text { Der Nachweis von XDR-Stämmen gelingt nur in sogenannten } \\
\text { Dritte-Welt-Ländern. }\end{array}$ \\
\hline $\mathrm{D}$ & Die Virusausscheidung beträgt bis zu 6 Monate. & $\mathrm{D}$ & Die Tuberkulose wird meist als Tröpfcheninfektion übertragen. \\
\hline $\mathrm{E}$ & Die Umweltstabilität ist gering. & $\mathrm{E}$ & Arbeitsbedingte Infektionen kommen nicht vor. \\
\hline 15. & Welche Angabe zu Adenoviren ist korrekt? & 20. & Welche Aussage zur Tuberkulose trifft nicht zu? \\
\hline A & $\begin{array}{l}\text { Adenoviren sind für ca. } 50 \% \text { der akuten Infektionen des } \\
\text { Respirationstraktes verantwortlich. }\end{array}$ & A & „MDR“ = multiresistente Tuberkulosebakterien \\
\hline B & Die Übertragung erfolgt meist parenteral. & $\mathrm{B}$ & „XDR“ = extrem resistente Tuberkulosestämme \\
\hline $\mathrm{C}$ & Die Inkubationszeit bei Atemwegsinfektionen beträgt 2-6 Tage. & $\mathrm{C}$ & XDR-Stämme wurden 2005 in Südafrika entdeckt. \\
\hline $\mathrm{D}$ & $\begin{array}{l}\text { Es erfolgt keine Ausscheidung des Virus über das } \\
\text { Rachensekret. }\end{array}$ & $\mathrm{D}$ & Im Jahr 2005 gab es schätzungsweise 424000 XDR-TB- Erkrankungen \\
\hline $\mathrm{E}$ & $\begin{array}{l}\text { Augeninfektionen können durch Adenoviren nicht verursacht } \\
\text { werden. }\end{array}$ & $\mathrm{E}$ & Im Jahr 2005 gab es schätzungsweise 424000 MDR-TB-Infektionen. \\
\hline
\end{tabular}




\section{CME-Fragen zu Respiratorische Erkrankungen: Antwortbogen Seite 1}

\section{A Angaben zur Person}

Bitte nur eine Antwort pro Frage ankreuzen

\begin{tabular}{l} 
Name, Vorname, Titel: \\
Straße, Hausnummer: \\
\hline
\end{tabular}

Anschrift: $\square$ privat $\square$ dienstlich

EFN-Nummer:

Ich bin Mitglied der Ärztekammer (bitte Namen der Kammer eintragen):

Jahr meiner Approbation:

Ich befinde mich in der Weiterbildung zum:

Ich habe eine abgeschlossene Weiterbildung in (bitte Fach eintragen):

Ich bin tätig als: $\square$ Assistenzarzt $\square$ Oberarzt $\quad \square$ Chefarzt $\square$ niedergelassener Arzt Sonstiges:

B Lernerfolgskontrolle

\begin{tabular}{|c|c|c|c|c|c|c|c|c|c|c|c|}
\hline 1 & A & B & C & D & $\mathbf{E}$ & 11 & A & B & C & D & $\mathbf{E}$ \\
\hline 2 & A & B & C & D & E & 12 & A & B & C & D & $E$ \\
\hline 3 & $\mathbf{A}$ & B & C & D & $\mathbf{E}$ & 13 & $\mathbf{A}$ & B & C & D & $\mathbf{E}$ \\
\hline 4 & A & B & C & D & $\mathbf{E}$ & 14 & A & B & C & D & $E$ \\
\hline 5 & A & B & C & D & $\mathbf{E}$ & 15 & A & B & C & D & $E$ \\
\hline 6 & $\mathbf{A}$ & B & C & D & $\mathbf{E}$ & 16 & A & B & C & D & $\mathbf{E}$ \\
\hline 7 & A & B & C & D & $\mathbf{E}$ & 17 & A & B & C & D & E \\
\hline 8 & A & B & C & D & $\mathbf{E}$ & 18 & A & B & C & D & $E$ \\
\hline 9 & A & B & C & D & $\mathbf{E}$ & 19 & A & B & C & D & $\mathbf{E}$ \\
\hline 10 & A & B & C & D & $\mathbf{E}$ & 20 & A & B & C & D & $\mathbf{E}$ \\
\hline
\end{tabular}

C Erklärung

Ich versichere, dass ich die Beantwortung der Fragen selbst und ohne Hilfe durchgeführt habe

Ort/Datum

D Feld für CME-Wertmarke

Bitte in dieses Feld die CME-Wertmarke kleben oder Ihre Abonnement-Nummer eintragen: (siehe Adressaufkleber)
Unterschrift

\begin{tabular}{l|l} 
E & Zertifizierungsfeld (wird durch den Verlag ausgefüllt)
\end{tabular} Ihr Ergebnis

Sie haben $\square$ von 20 Fragen richtig beantwortet.

Sie haben

$\square$ bestanden und 2 CME-Punkte erworben.

$\square$ nicht bestanden

Heidelberg, den

Datum

Stempel/Unterschrift

Bitte unbedingt Antwortbogen 2 ausfüllen! Bitte unbedingt Antwortbogen 2 ausfüllen! Bitte unbedingt Antwortbogen 2 ausfüllen! 
CME-Fragen zu Respiratorische Erkrankungen: Antwortbogen Seite 2

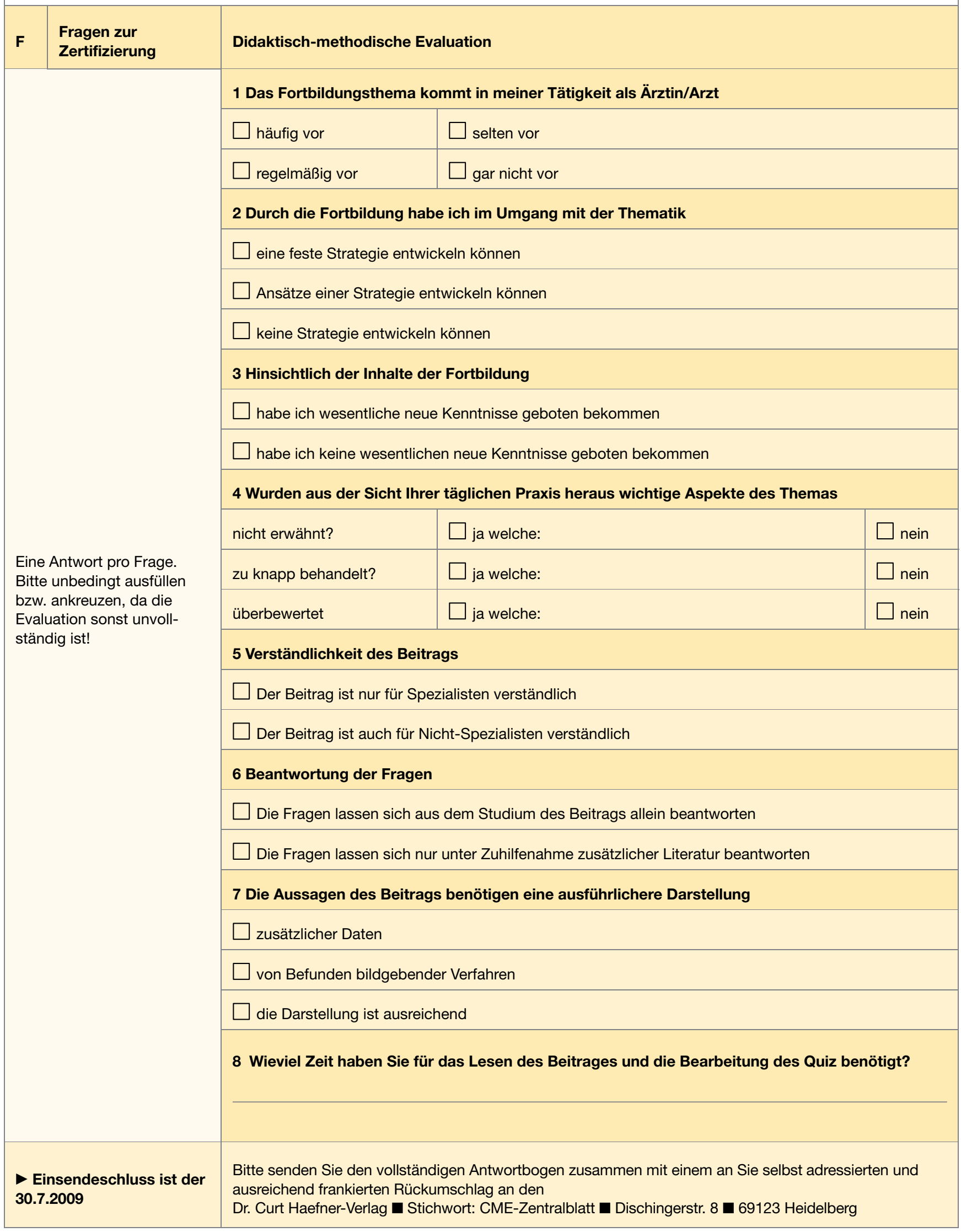

